\title{
A class of diffusion problem of Kirchhoff type with viscoelastic term involving the fractional Laplacian
}

Eugenio Cabanillas Lapa, Zacarias L. Huaringa Segura, Juan B. Bernui Barros and Eduardo V. Trujillo Flores

\begin{abstract}
This work is concerned with a class of diffusion problem of Kirchhoff type with viscoelastic term and nonlinear interior source in the setting of the fractional Laplacian. Under suitable conditions we prove the existence of global solutions and the exponential decay of the energy.
\end{abstract}

Mathematics Subject Classification (2010): 35K55, 35R11, 35B44, 35Q91.

Keywords: Kirchhoff-type diffusion problem, fractional Laplacian, local existence, Galerkin method.

\section{Introduction}

We consider the problem of finding $u=u(x, t)$ weak solutions to the following nonlinear heat equation of Kirchhoff type with variable exponent of nonlinearity, viscoelastic term and source term involving the fractional Laplacian

$$
\begin{aligned}
\left(1+a|u|^{r(x)-2}\right) u_{t}+ & M\left(\|u\|_{w_{0}}^{2}\right)(-\Delta)^{s} u-\int_{0}^{t} g(t-\tau)(-\Delta)^{s} u(\tau) d \tau \\
& \left.=|u|^{\rho-1} \quad \text { in } \Omega \times\right] 0, \infty[ \\
& u=0 \quad \text { in }\left(\mathbb{R}^{N} \backslash \Omega\right) \times[0, \infty[, \\
& u(x, 0)=u^{0}(x) \quad \text { in } \Omega,
\end{aligned}
$$

where $\Omega \subseteq \mathbb{R}^{N}$ is a smooth bounded domain, $\left.M(t)=t^{\alpha-1}+1, t \geq 0, s \in\right] 0,1[$, $2<\rho<2_{s}^{*}=\frac{2 N}{N-2 s}, 2<\frac{N}{s}, \alpha>1 ; g:\left[0, \infty[\rightarrow] 0, \infty\left[\right.\right.$ belongs to $C^{1}([0, \infty[), g(0)>0$, $l=1-\int_{0}^{\infty} g(\tau) d \tau>0, g^{\prime}(t) \leq 0$ and $r$ is a given continuous function.

This type of problems without viscoelastic term (that is $g=0), r(x)=$ constant and $M(t)=1$ have been considered by many authors with the standard Laplace operator 
544 E. Cabanillas L., Z.L. Huaringa S., J.B. Bernui B. and E.V. Trujillo F.

$(-\triangle)^{s}, s=1$ and can be seen as special case of doubly nonlinear parabolic type equations

$$
(\varphi(u))_{t}-\Delta u=f(u)
$$

which appear in the mathematical modelling of various physical processes such as flows of incompressible turbulent fluids or gases in pipes, processes of filtration in porous media, glaciology, see $[3,8,7,20,33,52]$ and the further references therein. When $a=0, M(t)=1$ and $s=1$, equation (1.1) is reduced to the following equation

$$
u_{t}-\Delta u+\int_{0}^{t} g(t-\tau) \Delta u(\tau) d \tau=f(u) .
$$

This equation arises from the study of heat conduction in materials with memory. The questions of solvability and the long time behavior of solutions of the abstract evolutions equations of type

$$
u_{t}-B u+\int_{0}^{t} g(t-\tau) A u(\tau) d \tau=f(u),
$$

where $A$ and $B$ are given operators, were studied in $[12,19,36,40]$.

Also, doubly nonlinear nonlocal parabolic equations

$$
(\varphi(u))_{t}-\operatorname{div} \sigma(\nabla u)=\int_{0}^{t} g(t-\tau) \operatorname{div} \sigma(\nabla u(\tau)) d \tau+f(x, t, u),
$$

were studied in $[9,30,47,48,49,50]$.

On the other hand, many fractional and nonlocal operators are actively studied in the recent years. This type of operators arises in a quite natural way in many interesting applications, such as, finance, physics, game theory, Lévy stable diffusion processes, crystal dislocation, one can see $[10,35,51]$ and their references. Some general motivations regarding the fractional Laplacian can be explicitly found in the recent monograph [17]. Nonlocal evolution equations of the form

$$
u_{t}=\int_{\mathbb{R}^{N}}(u(y, t)-u(x, t)) K(x-y) d y,
$$

and variations of it, have been widely used to model diffusion processes, more precisely as stated in [26], if $u(x, t)$ is thought as a density of population at the point $x$ at time $t$ and $K(x-y)$ is thought of as the probability distribution of jumping from location $y$ to location $x$, then $\int_{\mathbb{R}^{N}}(u(y, t) K(x-y)) d y$ is the rate at which individuals are arriving at position $x$ from all other places and $\int_{\mathbb{R}^{N}}(u(x, t) K(x-y)) d y$ is the rate which they are leaving location $x$ to travel to all other sites. So the density $u$ satisfies (1.3). For recent references on nonlocal diffusion problems, see [5, 1, 29]. If we consider the effects of total population, then equation (1.3) becomes

$u_{t}=M\left(\iint_{\mathbb{R}^{N}}|u(y, t)-u(x, t)|^{2} K(x-y) d x d y\right) \int_{\mathbb{R}^{N}}(u(y, t)-u(x, t)) K(x-y) d y$. 
In particular, if $s \rightarrow 1^{-}$and $K(x)=|x|^{-N-2 s}$, then equation reduces to

$$
u_{t}=-M\left(\iint_{\mathbb{R}^{N}}|\nabla u|^{2} d x\right) \Delta u,
$$

which is equation (1.2), with $M(t)=1, g(t)=0$ and $f(t)=0$. Thus it is natural to consider equation (1.1) as a generalization of the model (1.4). The main feature of the equation (1.1) is that contains an integrodifferential operator usually called memory term or viscoelastic term, which can be used to represent the damping or memory effect on the diffusion process.

The research on nonlinear problems with variable exponent growth conditions is an an attractive topic, and these problems have many applications in nonlinear elastic electrorheological fluids and image restoration, see [2, 16, 18, 53].

The study of Kirchhoff type problems has been receiving considerable attention in more recent years, see $[31,38,42,41]$. The interest arises from their contribution to the modeling of many physical and biological phenomena. We refer for example the reader to the bibliography $[4,6,11,32,37]$ and references therein. The first result concerning fractional Kirchhoff problems was obtained in Fiscella and Valdinoci [27]. In this paper, the fractional Kirchhoff equation was first introduced and motivated. In [42], by using the sub-differential approach, Pucci et al obtained the well-posedness of solutions for problem (1.1) with $f(x, t)$ instead of $|u|^{p-2} u$. Moreover, the large-time behavior and extinction of solutions also are considered. With the help of potential well theory, Fu and Pucci [28] studied the existence of global weak solutions and established the vacuum isolating and blow-up of strong solutions, provided that $M \equiv 1$ and $2<p \leq 2_{s}^{*}=2 N /(N-2 s)$. However, the Kirchhoff function $M$ is assumed to satisfy the non-degenerate condition in the above papers. In [41], Pan et al investigated for the first time the existence of global weak solutions for degenerate Kirchhoff-type diffusion problems involving fractional p-Laplacian, by combining the Galerkin method with potential well theory, for the special function $M(t)=t$; Mingqi et al. [38] proved the local existence and blow-up of solutions for the similar equation with more general conditions on $M$ which cover the degenerate case.

In the works mentioned above, there are few about the global existence and exponential decay rate for doubly nonlinear parabolic equation, involving variable exponent conditions, with viscoelastic term in the fractional setting. Motivated by it, we intend to study global existence for the problem (1.1) by using Galerkin's method and also give the exponential decay rate of the energy via the energy perturbation method. The plan of the paper is the following. In Section 2, we give the preliminaries for our research. In Section 3, by using the Galerkin approximation method we are able to prove global existence and finally, we obtain the exponential decay under certain class of initial data.

\section{Preliminaries}

In this section, we present some materials and assumptions needed in the rest of this paper. 
We denote: $Q=\mathbb{R}^{2 n} \backslash(\mathcal{C} \Omega \times \mathcal{C} \Omega)$ and $\mathcal{C} \Omega:=\mathbb{R}^{n} \backslash \Omega$,

$$
W=\left\{u: \mathbb{R}^{N} \rightarrow \mathbb{R}:\left.u\right|_{\Omega} \in L^{2}(\Omega), \iint_{Q} \frac{|u(x)-u(y)|^{2}}{|x-y|^{N+2 s}} d x d y<\infty\right\},
$$

where $\left.u\right|_{\Omega}$ represents the restriction to $\Omega$ of function $u(x)$. Also, we define the following linear subspace of $W$,

$$
W_{0}=\left\{u \in W: u=0 \text { a.e. in } \mathbb{R}^{N} \backslash \Omega\right\} .
$$

The linear space $W$ is endowed with the norm

$$
\|u\|_{W}:=\|u\|_{L^{2}(\Omega)}+\left(\iint_{Q} \frac{|u(x)-u(y)|^{2}}{|x-y|^{N+2 s}} d x d y\right)^{1 / 2} .
$$

It is easily seen that $\|\cdot\|_{W}$ is a norm on $W$ and $C_{0}^{\infty}(\Omega) \subseteq W_{0}$.

The functional

$$
\|u\|_{W_{0}}=\left(\iint_{Q} \frac{|u(x)-u(y)|^{2}}{|x-y|^{N+2 s}} d x d y\right)^{1 / 2}
$$

is a equivalent norm on $W_{0}=\left\{u \in W: u(x)=0\right.$ a.e. in $\left.\mathbb{R}^{N} \backslash \Omega\right\}$ which is a closed linear subspace of $W$. Furthermore $\left(W_{0},\|\cdot\|_{W_{0}}\right)$ is a Hilbert space with inner product

$$
\langle u, v\rangle_{W_{0}}=\iint_{Q} \frac{(u(x)-u(y))(v(x)-v(y))}{|x-y|^{N+2 s}} d x d y .
$$

We review the main embedding results for the space $W_{0}$.

Lemma 2.1 ([44, 43, 46, 45]). The embedding $W_{0} \hookrightarrow L^{r}(\Omega)$ is continuous for any $r \in\left[1,2_{s}^{*}\right]$, and compact for any $r \in\left[1,2_{s}^{*}[\right.$.

Lemma 2.2 ([39, Lemma 2.1]). Let $N \geq 1,0<s<1, p>1, q \geq 1, \tau>0$ and $0<\theta<1$ be such that $\frac{1}{\tau}=\theta\left(\frac{1}{p}-\frac{s}{N}\right)+\frac{1-\theta}{q}$ then

$$
\|u\|_{L^{\tau}\left(\mathbb{R}^{n}\right)} \leq\|u\|_{W^{s, p}\left(\mathbb{R}^{n}\right)}^{\theta}\|u\|_{L^{q}\left(\mathbb{R}^{n}\right)}^{1-\theta}, \quad \forall u \in C_{0}^{1}\left(\mathbb{R}^{N}\right) .
$$

Now, we recall some necessary background knowledge and propositions concerning the generalized Lebesgue-Sobolev spaces. We refer the reader to [21, 22, 25, 23] for details.

Set

$$
C_{+}(\bar{\Omega})=\{p(x): p(x) \in C(\bar{\Omega}), p(x)>1, \text { for all } x \in \bar{\Omega}\} .
$$

For any $p \in C_{+}(\bar{\Omega})$ we define

$$
\begin{gathered}
p^{+}=\max \{p(x): x \in \bar{\Omega}\}, \quad p^{-}=\min \{p(x) ; x \in \bar{\Omega}\} \\
L^{p(x)}(\Omega)=\left\{u: u \text { is a measurable real-valued function, } \int_{\Omega}|u(x)|^{p(x)} d x<\infty\right\},
\end{gathered}
$$


with the norm

$$
\|u\|_{p(x)} \equiv\|u\|_{L^{p(x)}(\Omega)}=\inf \left\{\lambda>0: \int_{\Omega}\left|\frac{u(x)}{\lambda}\right|^{p(x)} d x \leq 1\right\}
$$

becomes a Banach space [34]. We also define the space

$$
W^{1, p(x)}(\Omega)=\left\{u \in L^{p(x)}(\Omega):|\nabla u| \in L^{p(x)}(\Omega)\right\},
$$

equipped with the norm

$$
\|u\|_{W^{1, p(x)}(\Omega)}=\|u(x)\|_{p(x)}+\|\nabla u(x)\|_{p(x)} .
$$

We denote by $W_{0}^{1, p(x)}(\Omega)$ the closure of $C_{0}^{\infty}(\Omega)$ in $W^{1, p(x)}(\Omega)$. Of course the norm $\|u\|=\|\nabla u\|_{p(x)}$ is an equivalent norm in $W_{0}^{1, p(x)}(\Omega)$.

Proposition $2.3([24,25])$. (i) The conjugate space of $L^{p(x)}(\Omega)$ is $L^{p^{\prime}(x)}(\Omega)$, where

$$
\frac{1}{p(x)}+\frac{1}{p^{\prime}(x)}=1 \text {. }
$$

For any $u \in L^{p(x)}(\Omega)$ and $v \in L^{p^{\prime}(x)}(\Omega)$, we have

$$
\int_{\Omega}|u v| d x \leq\left(\frac{1}{p^{-}}+\frac{1}{p^{\prime-}}\right)\|u\|_{p(x)}\|v\|_{p^{\prime}(x)} \leq 2\|u\|_{p(x)}\|v\|_{p^{\prime}(x)} .
$$

(ii) If $p_{1}(x), p_{2}(x) \in C_{+}(\bar{\Omega})$ and $p_{1}(x) \leq p_{2}(x)$ for all $x \in \bar{\Omega}$, then $L^{p_{2}(x)}(\Omega) \hookrightarrow$ $L^{p_{1}(x)}(\Omega)$ and the embedding is continuous.

Proposition 2.4 ([25]). Set $\rho(u)=\int_{\Omega}|\nabla u(x)|^{p(x)} d x$, then for $u \in W_{0}^{1, p(x)}(\Omega)$ and $\left(u_{k}\right) \subset W_{0}^{1, p(x)}(\Omega)$, we have

(1) $\|u\|<1$ (respectively $=1 ;>1$ ) if and only if $\rho(u)<1$ (respectively $=1 ;>1$ );

(2) for $u \neq 0,\|u\|=\lambda$ if and only if $\rho\left(\frac{u}{\lambda}\right)=1$;

(3) if $\|u\|>1$, then $\|u\|^{p^{-}} \leq \rho(u) \leq\|u\|^{p^{+}}$;

(4) if $\|u\|<1$, then $\|u\|^{p^{+}} \leq \rho(u) \leq\|u\|^{p^{-}}$;

(5) $\left\|u_{k}\right\| \rightarrow 0$ (respectively $\rightarrow \infty$ ) if and only if $\rho\left(u_{k}\right) \rightarrow 0$ (respectively $\rightarrow \infty$ ).

For $x \in \Omega$, let us define

$$
p^{*}(x)= \begin{cases}\frac{N p(x)}{N-p(x)} & \text { if } p(x)<N \\ \infty & \text { if } p(x) \geq N\end{cases}
$$

Proposition 2.5 ([23]). If $q \in C_{+}(\bar{\Omega})$ and $q(x) \leq p^{*}(x)\left(q(x)<p^{*}(x)\right)$ for $x \in \bar{\Omega}$, then there is a continuous (compact) embedding $W^{1, p(x)}(\Omega) \hookrightarrow L^{q(x)}(\Omega)$.

Lemma 2.6. Let $2<r<\rho<2_{s}^{*}$.For each $\varepsilon>0$, there exists a positive constant $C_{\varepsilon}$ such that

$$
\|v\|_{\rho}^{\rho} \leq \varepsilon\|v\|_{W_{0}}^{2}+C_{\varepsilon}\|v\|_{r}^{k r},
$$

for all $v \in W_{0} \cap L^{r}(\Omega)$ where

$$
k=\frac{2 \rho(1-\theta)}{r(2-\rho \theta)} \quad, \quad \theta=\left(\frac{1}{r}-\frac{1}{\rho}\right)\left(\frac{s}{N}-\frac{1}{2}+\frac{1}{r}\right)^{-1} .
$$


548 E. Cabanillas L., Z.L. Huaringa S., J.B. Bernui B. and E.V. Trujillo F.

Proof. The conclusion of lemma immediately follows from Lemma 2.2 and Young's inequality.

Lemma 2.7. [34, Theorem 1, pag 23] Suppose that

$$
r \in L_{+}^{\infty}(\Omega), r^{-} \geq 2, w \in L^{r(x)}(\Omega \times] 0, T[) \text { and } \frac{\partial}{\partial t}\left(|w|^{r(x)-2} w\right) \in L^{r^{\prime}(x)}(\Omega \times] 0, T[) .
$$

Then, for any $s, \tau \in[0, T], s<\tau$ the following formula of integration by parts is correct:

$$
\int_{s}^{\tau} \int_{\Omega} w\left(\frac{1}{r(x)-1}|w|^{r(x)-2} w\right) d x d t=\int_{\Omega} \frac{1}{r(x)}|w(\tau)|^{r(x)} d x-\int_{\Omega} \frac{1}{r(x)}|w(s)|^{r(x)} d x .
$$

\section{Global existence and exponential decay}

In this section, we focus our attention on the global existence and exponential decay of the solution to problem (1.1).

Definition 3.1. Let $T>0$. A weak solution of problem (1.1) is a function $u \in$ $L^{\infty}\left(0, T ; W_{0}\right)$, with $u_{t} \in L^{2}\left(0, T ; L^{2}(\Omega)\right)$ and $\left(|u|^{r(x) / 2}\right)_{t} \in L^{2}(\Omega \times] 0, T[)$ such that

$$
\begin{array}{r}
\int_{0}^{T} \int_{\Omega}\left(1+a|u|^{r(x)-2}\right) u_{t} w d x d t+M\left(\|u\|_{w_{0}}^{2}\right) \int_{0}^{T}\langle u, w\rangle_{W_{0}} d t \\
\quad-\int_{0}^{T} \int_{0}^{t} g(t-\tau)\langle u(\tau), w\rangle_{W_{0}} d \tau d t=\int_{0}^{T} \int_{\Omega}|u|^{\rho-1} w d x d t
\end{array}
$$

for all $w \in L^{2}\left(0, T ; W_{0}\right)$, and $u(x, 0)=u^{0}(x) \in W_{0}$.

Theorem 3.2 (Local Solution). Assume $u^{0} \in W_{0}, 2<r^{-}<\rho<2_{s}^{*}, \rho<2+\frac{2 r s}{N}$, $\left.r^{+} \in\right] 2,2_{s}^{*}[$, then problem (1.1) has a unique weak solution $u$ for $T$ small enough.

Proof. We prove the local existence of weak solutions by using the Faedo-Galerkin method benefited from the ideas of [14]. We choose a sequence $\left\{w_{\nu}\right\}_{\nu \in \mathbb{N}} \subseteq C_{0}^{\infty}(\Omega)$ such that $C_{0}^{\infty}(\Omega) \subseteq \bigcup_{\nu=1}^{\infty} V_{m} \quad$ and $\left\{w_{\nu}\right\}$ is a standard orthonormal basis with respect to the Hilbert space $L^{2}(\Omega)$ and an orthogonal basis in $W_{0}$, where

$$
V_{m}=\operatorname{spam}\left\{w_{1}, w_{2}, \ldots w_{m}\right\} .
$$

Now, we construct approximate solutions $u_{m}(m=1,2, \cdots)$, of the problem (1.1), in the form

$$
u_{m}(x, t)=\sum_{i=1}^{m} g_{j m}(t) w_{j}(x)
$$


where the coefficient functions $g_{j m}$ satisfy the system of ordinary differential equations

$$
\begin{gathered}
\int_{\Omega}\left(1+a\left|u_{m}(t)\right|^{r(x)-2}\right) u_{m t}(t) w_{j} d x+M\left(\left\|u_{m}(t)\right\|_{w_{0}}^{2}\right)\left\langle u_{m}(t), w_{j}\right\rangle_{W_{0}} \\
-\int_{0}^{t} g(t-\tau)\left\langle u_{m}(\tau), w_{j}\right\rangle_{W_{0}} d \tau d t=\int_{\Omega}\left|u_{m}(t)\right|^{\rho-1} w_{j} d x \\
j=1,2, \cdots m . \\
u_{m}(x, 0)=u_{m}^{0}(x) \rightarrow u^{0}(x) \quad \text { in } W_{0} .
\end{gathered}
$$

Let us show that the system (3.1) is locally solvable.

It is clear that (3.1) can be rewritten in the form

$$
\frac{d}{d t} \Phi\left(g_{m}(t)\right)=-M\left(\left\|\sum_{i=1}^{m} g_{j m}(t) w_{j}(x)\right\|_{W_{0}}^{2}\right) B g_{m}(t)+\int_{0}^{t} g(t-\tau) B g_{m}(\tau) d \tau+F\left(g_{m}(t)\right)
$$

where

$$
\begin{aligned}
& g_{m}(t)=\left(g_{m 1}(t), g_{m 2}(t), \cdots, g_{m m}(t)\right)^{t}, \quad B=\left[\left\langle w_{i}, w_{j}\right\rangle\right]_{1 \leq i, j \leq m}, \\
& \Phi(\eta)=\left(\Phi_{1}(\eta), \Phi_{2}(\eta), \cdots, \Phi_{m}(\eta)\right)^{t} \quad \text { with } \eta=\left(\eta_{1}, \eta_{2}, \cdots, \eta_{m}\right) \in \mathbb{R}^{m}, \\
& \Phi_{i}(\eta)=\int_{\Omega}\left\{\sum_{j=1}^{m} \eta_{j} w_{j}+\frac{a}{r(x)-1}\left|\sum_{k=1}^{m} \eta_{k} w_{k}\right|^{r(x)-2} \sum_{k=1}^{m} \eta_{k} w_{k}\right\} w_{i} d x \quad i=1,2, \cdots, m
\end{aligned}
$$

and

$F(\eta)=\left(\int_{\Omega}\left|\sum_{k=1}^{m} \eta_{j} w_{j}\right|^{\rho-1} w_{1} d x, \int_{\Omega}\left|\sum_{k=1}^{m} \eta_{j} w_{j}\right|^{\rho-1} w_{2} d x, \cdots, \int_{\Omega}\left|\sum_{k=1}^{m} \eta_{j} w_{j}\right|^{\rho-1} w_{m} d x\right)^{t}$.

This system is equivalent to

$$
\begin{aligned}
& \Phi\left(g_{m}(t)\right)=\Phi\left(g_{m}(0)\right) \\
& +\int_{0}^{t}\left[-M\left(\left\|\sum_{i=1}^{m} g_{j m}(t) w_{j}(x)\right\|_{W_{0}}^{2}\right) B g_{m}(t)+\int_{0}^{\xi} g(\xi-\tau) B g_{m}(\tau) d \tau+F\left(g_{m}(\xi)\right)\right] d \xi .
\end{aligned}
$$

If $\zeta, \eta$ are to arbitrary elements of $\mathbb{R}^{m}$, we get

$$
(\Phi(\zeta)-\Phi(\eta), \zeta-\eta)_{\mathbb{R}^{m}} \geq C_{m}|\zeta-\eta|_{\mathbb{R}^{m}}^{2}
$$

here $C_{m}$ is a constant such that, for any $g_{m}$ in $\mathbb{R}^{m}$

$$
\int_{\Omega}\left|u_{m}\right|^{2} d x \geq C_{m}\left|g_{m}\right|_{\mathbb{R}^{m}}^{2}
$$

Then $\Phi$ is monotone coercive. Also it is obviously continuous. So, by the Brouwer theorem $\Phi$ is onto. In view of $(3.3), \Phi^{-1}$ is locally Lipchitz continuous. 
Consider the map $L: C\left(0, T, \mathbb{R}^{m}\right) \rightarrow C\left(0, T, \mathbb{R}^{m}\right)$, defined by

$$
\begin{aligned}
& L\left(g_{m}\right)(t)=\Phi^{-1}\left(\Phi\left(g_{m}(0)\right)\right. \\
& \left.+\int_{0}^{t}\left[-M\left(\left\|\sum_{i=1}^{m} g_{j m}(t) w_{j}(x)\right\|_{W_{0}}^{2}\right) B g_{m}(t)+\int_{0}^{\xi} g(\xi-\tau) B g_{m}(\tau) d \tau+F\left(g_{m}(\xi)\right)\right] d \xi\right), \\
& t \in[0, T] .
\end{aligned}
$$

It is not hard to prove that $L$ is completely continuous and also, there exist (sufficient small) $T_{m}>0$ and (sufficient large) $R>0$ such that $L\left(\overline{B_{R}}\right) \subseteq \overline{B_{R}}$, where $\overline{B_{R}}$ is the ball in $C\left(0, T_{m}, \mathbb{R}^{m}\right)$ with center the origin and radius $R$. Consequently, by Schauder's theorem, the operator $L$ has a fixed point in $C\left(0, T_{m}, \mathbb{R}^{m}\right)$. This fixed point is a solution of (3.2).

So, we can obtain an approximate solution $u_{m}(t)$ of $(3.1)$ in $V_{m}$ over $\left[0, T_{m}\right.$ [ and it can be extended to the whole interval $[0, T]$, for all $T>0$, as a consequence of the a priori estimates that shall be proven in the next step.

\section{The First Estimate}

Multiplying (3.1) by $g_{j m}(t)$ and adding in $j=1 ; \cdots ; m$, we have

$$
\begin{gathered}
\int_{\Omega}\left(1+a\left|u_{m}(t)\right|^{r(x)-2}\right) u_{m t}(t) u_{m}(t) d x+M\left(\left\|u_{m}(t)\right\|_{w_{0}}^{2}\right)\left\langle u_{m}(t), u_{m}(t)\right\rangle_{W_{0}} \\
-\int_{0}^{t} g(t-\tau)\left\langle u_{m}(\tau), u_{m}(t)\right\rangle_{W_{0}} d \tau d t=\int_{\Omega}\left|u_{m}(t)\right|^{\rho-1} u_{m}(t) d x
\end{gathered}
$$

which implies, integrating with respect to the time variable from 0 to $t$ on both sides, using Lemma 2.7 that

$$
\begin{array}{r}
S_{m}(t)=S_{m}(0)+\int_{0}^{t} d \lambda \int_{0}^{\lambda} g(\lambda-\tau)\left\langle u_{m}(\tau), u_{m}(\lambda)\right\rangle_{W_{0}} d \tau \\
+\int_{0}^{t} \int_{\Omega}\left|u_{m}(t)\right|^{\rho-1} u_{m}(\tau) d x d \tau
\end{array}
$$

where

$S_{m}(t)=\int_{\Omega}\left|u_{m}(t)\right|^{2} d x+a \int_{\Omega} \frac{1}{r(x)}\left|u_{m}(t)\right|^{r(x)} d x+\int_{0}^{t}\left(\left\|u_{m}(\tau)\right\|_{W_{0}}^{2 \alpha}+\left\|u_{m}(\tau)\right\|_{W_{0}}^{2}\right) d \tau$.

Let us introduce the function $\Theta(\lambda)=\int_{0}^{\lambda} g(\lambda-\tau)\left\|u_{m}(\tau)\right\|_{W_{0}}$. Estimating the second term on right-hand side of (3.5) we have

$$
\begin{aligned}
\int_{0}^{t} d \lambda \int_{0}^{\lambda} g(\lambda-\tau)\left\langle u_{m}(\tau), u_{m}(\lambda)\right\rangle_{W_{0}} d \tau \leq & \frac{1}{2} \int_{0}^{t}\left(\left\|u_{m}(\tau)\right\|_{W_{0}}^{2 \alpha}+\left\|u_{m}(\tau)\right\|_{W_{0}}^{2}\right) d \tau \\
& +\frac{1}{2} \int_{0}^{t} \Theta^{2}(\lambda) d \lambda
\end{aligned}
$$


But, using Young Inequality and noting that $\int_{0}^{\infty} g(\tau) d \tau<1$, we get

$$
\int_{0}^{t} \Theta^{2}(\lambda) d \lambda \leq \int_{0}^{\infty} g(\tau) d \tau \int_{0}^{t}\left(\left\|u_{m}(\tau)\right\|_{W_{0}}^{2 \alpha}+\left\|u_{m}(\tau)\right\|_{W_{0}}^{2}\right) d \tau .
$$

Plugging (3.6)- (3.7)into (3.5), it follows that

$$
\begin{aligned}
S_{m}(t) \leq S_{m}(0) & +\frac{1}{2}\left(1+\int_{0}^{\infty} g(\tau) d \tau\right) \int_{0}^{t}\left(\left\|u_{m}(\tau)\right\|_{W_{0}}^{2 \alpha}+\left\|u_{m}(\tau)\right\|_{W_{0}}^{2}\right) d \tau \\
& +\int_{0}^{t}\left\|u_{m}(t)\right\|_{\rho}^{\rho} d \tau .
\end{aligned}
$$

To estimate the last term in (3.8) we use Lemma 2.6,

$$
\int_{0}^{t}\left\|u_{m}(t)\right\|_{\rho}^{\rho} d \tau \leq \varepsilon \int_{0}^{t}\left(\left\|u_{m}(\tau)\right\|_{W_{0}}^{2 \alpha}+\left\|u_{m}(\tau)\right\|_{W_{0}}^{2}\right) d \tau+c_{0} \int_{0}^{t} S_{m}^{k}(\lambda) d \lambda,
$$

where $k=\frac{2 \rho(1-\theta)}{r^{-}(2-\rho \theta)}>1$. Taking $\varepsilon$ suitably small in (3.9), it follows from (3.5)-(3.9) that

$$
S_{m}(t) \leq \hat{C}_{0}+\hat{C}_{1} \int_{0}^{t} S_{m}^{k}(\lambda) d \lambda .
$$

Hence, by employing Bihari-Langenhop's inequality (cf. [13]), there exists a constant $T_{0}$ such that

$$
S_{m}(t) \leq C_{T_{0}}, \quad \forall t \in\left[0, T_{0}\right]
$$

\section{The Second Estimate}

Multiplying (3.1) by $g_{j m}^{\prime}(t)$ and adding in $j=1 ; \cdots ; m$, it holds that

$$
\begin{aligned}
\frac{d}{d t}\left\{\frac{1}{2 \alpha}\left\|u_{m}(t)\right\|_{W_{0}}^{2 \alpha}+\frac{1}{2}\left(1-\int_{0}^{t} g(\tau) d \tau\right)\left\|u_{m}(t)\right\|_{W_{0}}^{2}+\frac{1}{2}(g \diamond u)(t)\right. \\
\left.-\frac{1}{\rho} \int_{\Omega}\left|u_{m}(t)\right|^{\rho-1} u_{m}(t) d x\right\}+\left\|u_{m t}(t)\right\|_{2}^{2}+a \int_{\Omega}\left|u_{m}(t)\right|^{r(x)-2}\left|u_{m t}(t)\right|^{2} d x \\
=\frac{1}{2}\left(g^{\prime} \diamond u\right)(t)-\frac{1}{2} g(t)\left\|u_{m}(t)\right\|_{W_{0}}^{2} .
\end{aligned}
$$

where $(g \diamond u)(t)=\int_{0}^{t} g(t-\tau)\|u(t)-u(\tau)\|_{W_{0}}^{2} d \tau$.

Integrating $(3.12)$ on $[0, t], t \leq T_{0}$ we get

$$
\begin{aligned}
\int_{0}^{t}\left\|u_{m t}(t)\right\|_{2}^{2} & +a \int_{0}^{t} \int_{\Omega}\left|u_{m}(t)\right|^{r(x)-2}\left|u_{m t}(t)\right|^{2} d x+\frac{1}{2 \alpha}\left\|u_{m}(t)\right\|_{W_{0}}^{2 \alpha}+\frac{l}{2}\left\|u_{m}(t)\right\|_{W_{0}}^{2} \\
& \leq \frac{1}{2 \alpha}\left\|u_{m}(0)\right\|_{W_{0}}^{2 \alpha}+\left.\frac{1}{2}\left\|u_{m}(0)\right\|\right|_{W_{0}} ^{2}-\frac{1}{\rho} \int_{\Omega}\left|u_{m}(0)\right|^{\rho-1} u_{m}(0) d x \\
& +\frac{1}{\rho} \int_{\Omega}\left|u_{m}(t)\right|^{\rho-1} u_{m}(t) d x .
\end{aligned}
$$


From the assumptions on $\rho$ and $u^{0}$, Lemma 2.6 and the estimate (3.11), it follows that

$$
\int_{0}^{t}\left\|u_{m t}(t)\right\|_{2}^{2}+a \int_{0}^{t} \int_{\Omega}\left|u_{m}(t)\right|^{r(x)-2}\left|u_{m t}(t)\right|^{2} d x+\frac{1}{2 \alpha}\left\|u_{m}(t)\right\|_{W_{0}}^{2 \alpha}+\frac{l}{2}\left\|u_{m}(t)\right\|_{W_{0}}^{2}
$$

for some constant $M_{1}>0$.

By the above estimates (3.11) and (3.13), $\left\{u_{m}\right\}$ have subsequences still denoted by $\left\{u_{m}\right\}$ such that

$$
\begin{gathered}
u_{m} \rightarrow u \quad \text { weakly* in } L^{\infty}\left(0, T_{0} ; W_{0}\right), \\
u_{m t} \rightarrow u_{t} \quad \text { weakly in } L^{2}\left(0, T_{0} ; L^{2}(\Omega)\right), \\
\left(\left|u_{m}\right|^{r(x) / 2}\right)_{t} \rightarrow \chi \quad \text { weakly in } L^{2}\left(0, T_{0} ; L^{2}(\Omega)\right) .
\end{gathered}
$$

Employing the same arguments as in [16] we can prove that

$$
\begin{gathered}
\chi=\left(|u|^{r(x) / 2}\right)_{t}\left|u_{m}\right|^{r(x) / 2} u_{m t} \rightarrow|u|^{r(x) / 2} u_{t} \text { weakly in } L^{2}(\Omega \times] 0, T_{0}[), \\
\left|u_{m}\right|^{\rho-1} \rightarrow|u|^{\rho-1} \quad \text { weakly in } L^{\frac{\rho}{\rho-1}}(\Omega \times] 0, T_{0}[) .
\end{gathered}
$$

Therefore, passing to the limit in (3.1) as $m \rightarrow+\infty$, by (3.14)- (3.18), we can show that $u$ satisfies the initial condition $u(0)=u^{0}$ and

$$
\begin{array}{r}
\int_{0}^{T} \int_{\Omega}\left(1+a|u|^{r(x)-2}\right) u_{t} w d x d t+M\left(\|u\|_{w_{0}}^{2}\right) \int_{0}^{T}\langle u, w\rangle_{W_{0}} d t \\
\quad-\int_{0}^{T} \int_{0}^{t} g(t-\tau)\langle u(\tau), w\rangle_{W_{0}} d \tau d t=\int_{0}^{T} \int_{\Omega}|u|^{\rho-1} w d x d t
\end{array}
$$

for all $w \in L^{2}\left(0, T_{0} ; W_{0}\right)$.

The uniqueness property of a solutions can be derived from [20, Theorem 3, p. 1095], observing that $\left(u+\frac{a}{r(x)-1}|u|^{r(x)-2} u\right) \in L^{2}(\Omega \times] 0, T_{0}[)$ and $A u=M\left(\|u\|_{w_{0}}^{2}\right)(-\Delta)^{s} u$ is a monotone operator. We omit the details.

Next, we consider the global existence and energy decay of solutions for problem (1.1). For this purpose we define the energy associated with problem (1.1) by

$$
E(t)=\frac{1}{2 \alpha}\|u(t)\|_{W_{0}}^{2 \alpha}+\frac{1}{2}\left(1-\int_{0}^{t} g(\tau) d \tau\right)\|u(t)\|_{W_{0}}^{2}+\frac{1}{2}(g \diamond u)(t)-\frac{1}{\rho} \int_{\Omega}|u(t)|^{\rho-1} u(t) d x .
$$

Then, we easily can check that

$$
\begin{aligned}
\frac{d}{d t} E(t)= & \frac{1}{2}\left(g^{\prime} \diamond u\right)(t)-\frac{1}{2} g(t)\|u(t)\|_{W_{0}}^{2}-\left\|u_{t}(t)\right\|_{2}^{2}-a \int_{\Omega}|u(t)|^{r(x)-2} u_{t}^{2}(t) d x \\
& \leq 0
\end{aligned}
$$

for any regular solution. This remains valid for weak solutions by simple density argument. This shows that $E(t)$ is a nonincreasing function.

Let $C_{*}$ be the optimal constant satisfying the Sobolev inequality $\|u\|_{\rho} \leq C_{*}\|u\|_{W_{0}}$, 
and $B_{1}=\frac{C_{*}}{\sqrt{l}}$. We define the function $h(\lambda)=\frac{1}{2} \lambda^{2}-\frac{B_{1}^{\rho}}{\rho} \lambda^{\rho}$. Then, we can verify that the function $h$ is increasing in $] 0, \lambda_{1}$ [, decreasing in $] \lambda_{1}, \infty[, h(\lambda) \rightarrow-\infty$, as $\lambda \rightarrow \infty$ and $h$ has a maximum at $\lambda_{1}$ with the maximum value

$$
h\left(\lambda_{1}\right)=E_{1}=\left(\frac{1}{2}-\frac{1}{\rho}\right) B_{1}^{-\frac{2 \rho}{\rho-2}}=\frac{\rho-2}{2 \rho} B_{1}^{-\frac{2 \rho}{\rho-2}} .
$$

where $\lambda_{1}$ is the first positive zero of the derivative function $h^{\prime}(\lambda)$.

Here, note that

$$
\begin{aligned}
E(t) & \geq \frac{l}{2}\|u(t)\|_{W_{0}}^{2}+\frac{1}{2}(g \diamond u)(t)-\frac{1}{\rho}\|u(t)\|_{\rho}^{\rho} \\
& \geq \frac{1}{2}\left(l\|u(t)\|_{W_{0}}^{2}+(g \diamond u)(t)\right)-\frac{B_{1}^{\rho} l^{\rho / 2}}{\rho}\|u(t)\|_{W_{0}}^{\rho} \\
& \geq h\left(\sqrt{l\|u(t)\|_{W_{0}}^{2}+(g \diamond u)(t)}\right), \forall t \geq 0 .
\end{aligned}
$$

Now, we are ready to state our result.

Theorem 3.3. Assume that hypotheses of Theorem 3.2 are satisfied. Consider $u_{0} \in W_{0}$, satisfying

$$
\begin{gathered}
0<l^{1 / 2}\left\|u_{0}\right\|_{W_{0}}<\lambda_{1} \\
\frac{1}{2 \alpha}\left\|u^{0}\right\|_{W_{0}}^{2 \alpha}+\frac{1}{2}\left\|u^{0}\right\|_{W_{0}}^{2}-\frac{1}{\rho} \int_{\Omega}\left|u^{0}\right|^{\rho-1} u^{0} d x<\left(\frac{\rho-2}{2 \rho}\right) B_{1}^{-\frac{2 \rho}{\rho-2}} .
\end{gathered}
$$

Then problem admits a global weak solution in time. In addition, if there exists a constant $\xi_{0}>0$ such that $g^{\prime}(t) \leq-\xi_{0} g(t)$, then this solution satisfies

$$
E(t) \leq L_{0} e^{-\gamma t}, \forall t \geq 0
$$

where $L_{0}$ and $\gamma$ are positive constants.

Proof. We will get global estimates for $u_{m}(t)$ solution of the approximate system (3.1) under the conditions (3.21)-(3.22) for $u^{0}$. For this, it suffices to show that

$$
E_{m}(t)+\int_{0}^{t}\left\|u_{m t}(t)\right\|_{2}^{2}+a \int_{0}^{t} \int_{\Omega}\left|u_{m}(t)\right|^{r(x)-2}\left|u_{m t}(t)\right|^{2} d x,
$$

where $E_{m}(t)$ is defined in (3.19) with $u(t)$ replaced by $u_{m}(t)$, is bounded and independently of $t$. From (3.12) and the definition of energy, we have

$$
E_{m}(t)+\int_{0}^{t}\left\|u_{m t}(t)\right\|_{2}^{2}+a \int_{0}^{t} \int_{\Omega}\left|u_{m}(t)\right|^{r(x)-2}\left|u_{m t}(t)\right|^{2} d x \leq E_{m}(0) .
$$

Due to convergence $u_{0 m} \rightarrow u^{0}$ in $W_{0}$ we see that $E_{m}(0)<\left(\frac{\rho-2}{2 \rho}\right) B_{1}^{-\frac{2 \rho}{\rho-2}}$ for sufficiently large $m$. We claim that there exists an integer $\nu_{0}$ such that

$$
\sqrt{l\left\|u_{m}(t)\right\|_{W_{0}}^{2}+\left(g \diamond u_{m}\right)(t)}<\lambda_{1} \quad \forall t \in\left[0, T_{m}\left[, m \geq \nu_{0} .\right.\right.
$$


554 E. Cabanillas L., Z.L. Huaringa S., J.B. Bernui B. and E.V. Trujillo F.

Suppose the claim is proved. Then $h\left(\sqrt{l\left\|u_{m}(t)\right\|_{W_{0}}^{2}+\left(g \diamond u_{m}\right)(t)}\right) \geq 0$ and from (3.20), (3.24)-(3.25) we get

$$
\left\|u_{m}(t)\right\|_{W_{0}}^{2 \alpha}+\left\|u_{m}(t)\right\|_{W_{0}}^{2}+\int_{0}^{t}\left\|u_{m t}(t)\right\|_{2}^{2}+a \int_{0}^{t} \int_{\Omega}\left|u_{m}(t)\right|^{r(x)-2}\left|u_{m t}(t)\right|^{2} d x \leq C .
$$

where $C$ is a constant independent of $m$.Thus, we obtain the global existence.

Proof of Claim: Suppose (3.25) is not true. Thus, for each $m>\nu_{0}$, there exists $t_{1} \in$ $\left[0, T_{m}[\right.$ such that

$$
\sqrt{l\left\|u_{m}\left(t_{1}\right)\right\|_{W_{0}}^{2}+\left(g \diamond u_{m}\right)\left(t_{1}\right)} \geq \lambda_{1} .
$$

Here, we observe that, from (3.21) and the convergence $u_{0 m} \rightarrow u^{0}$ in $W_{0}$ there exists $\nu_{1}$ such that

$$
l^{1 / 2}\left\|u_{m}(0)\right\|_{W_{0}}<\lambda_{1} \quad \forall m>\nu_{1} .
$$

Hence, by continuity there exists

$$
t^{*}=\inf \left\{t \in \left[0, T_{m}\left[: \sqrt{l\left\|u_{m}(t)\right\|_{W_{0}}^{2}+\left(g \diamond u_{m}\right)(t)} \geq \lambda_{1}\right\},\right.\right.
$$

such that

$$
\sqrt{l\left\|u_{m}\left(t^{*}\right)\right\|_{W_{0}}^{2}+\left(g \diamond u_{m}\right)\left(t^{*}\right)}=\lambda_{1} .
$$

By (3.20), we see that

$$
E_{m}\left(t^{*}\right) \geq h\left(\sqrt{l\left\|u_{m}\left(t^{*}\right)\right\|_{W_{0}}^{2}+\left(g \diamond u_{m}\right)\left(t^{*}\right)}\right)=h\left(\lambda_{1}\right)=E_{1}
$$

which contradicts $E_{m}(t) \leq E_{m}(0)<E_{1}, \forall t \geq 0$. Therefore our claim is true.

The above estimates permit us to pass to the limit in the approximate equation.

To show the uniform decay of the solution we introduce the perturbed energy functional

$$
F(t)=E(t)+\varepsilon \Phi(t)
$$

where $\varepsilon$ is a positive constant which shall be determined later, and

$$
\Phi(t)=\int_{\Omega}\left(|u|^{2}+\frac{a}{r(x)}|u|^{r(x)}\right) d x .
$$

It is straightforward to see that $F(t)$ and $E(t)$ are equivalent in the sense that there exist two positive constants $\beta_{1}$ and $\beta_{2}$ depending on $\varepsilon$ such that for $t \geq 0$

$$
\beta_{1} E(t) \leq F(t) \leq \beta_{2} E(t) .
$$

By taking the time derivative of the function $F$ defined above in (3.30), using (3.20), and performing several integration by parts, we get

$$
\begin{gathered}
\frac{d}{d t} F(t)=\frac{1}{2}\left(g^{\prime} \diamond u\right)(t)-\frac{1}{2} g(t)\|u(t)\|_{W_{0}}^{2}-\left\|u_{t}(t)\right\|_{2}^{2}-a \int_{\Omega}|u(t)|^{r(x)-2} u_{t}^{2}(t) d x \\
-\varepsilon\|u(t)\|_{W_{0}}^{2 \alpha}-\varepsilon\|u(t)\|_{W_{0}}^{2}+\varepsilon \int_{\Omega}|u(t)|^{\rho-1} u(t) d x+\varepsilon \int_{0}^{t} g(t-\tau)\langle u(\tau), u(t)\rangle_{W_{0}} d \tau .
\end{gathered}
$$


On the other hand, we can easily see that the condition $E(0)<E_{1}$ is equivalent to the inequality:

$$
B_{1}^{\rho}\left(\frac{2 \rho}{\rho-2} E(0)\right)^{\frac{\rho-2}{2}}<1 .
$$

From the assumption (3.21)-(3.22) and (3.24) we have

$$
\begin{aligned}
l\|u(t)\|_{W_{0}}^{2} & \leq\left(1-\int_{0}^{t} g(\tau) d \tau\right)\|u(t)\|_{W_{0}}^{2}+(g \diamond u)(t) \\
& <\lambda_{1}^{2}=B_{1}^{-\frac{2 \rho}{\rho-2}}
\end{aligned}
$$

which implies that

$$
\begin{aligned}
I(t) & =\left(1-\int_{0}^{t} g(\tau) d \tau\right)\|u(t)\|_{W_{0}}^{2}+(g \diamond u)(t)-\int_{\Omega}|u(t)|^{\rho-1} u(t) d x \\
& \geq l\|u(t)\|_{W_{0}}^{2}+(g \diamond u)(t)-\|u(t)\|_{\rho}^{\rho} \\
& \geq l\|u(t)\|_{W_{0}}^{2}-C_{*}^{\rho}\|u(t)\|_{W_{0}}^{\rho} \geq 0 .
\end{aligned}
$$

So, we have

$$
\begin{aligned}
\left(\frac{\rho-2}{2 \rho}\right) l\|u(t)\|_{W_{0}}^{2} & \leq \frac{\rho-2}{2 \rho}\left(1-\int_{0}^{t} g(\tau) d \tau\right)\|u(t)\|_{W_{0}}^{2} \\
& \leq \frac{\rho-2}{2 \rho}\left[\left(1-\int_{0}^{t} g(\tau) d \tau\right)\|u(t)\|_{W_{0}}^{2}+(g \diamond u)(t)\right]+\frac{1}{\rho} I(t) \\
& \leq E(t) \leq E(0),
\end{aligned}
$$

then

$$
l\|u(t)\|_{W_{0}}^{2} \leq \frac{2 \rho}{\rho-2} E(0) .
$$

Using the above inequality, we can deduce that

$$
\begin{aligned}
\left.\left|\int_{\Omega}\right| u\right|^{\rho-1} u \mid & \leq\|u(t)\|_{\rho}^{\rho} \\
& \leq C_{*}^{\rho}\|u(t)\|_{W_{0}}^{\rho} \frac{C_{*}^{\rho}}{l}\left(\frac{2 \rho}{l(\rho-2)} E(0)\right)^{\frac{\rho-2}{2}} l\|u(t)\|_{W_{0}}^{2} \\
& \equiv \theta l\|u(t)\|_{W_{0}}^{2} .
\end{aligned}
$$

From the Young inequality and the fact that

$$
\int_{0}^{t} g(\tau) d \tau \leq \int_{0}^{\infty} g(\tau) d \tau=1-l
$$


it follows that

$$
\begin{aligned}
& \int_{0}^{t} g(t-\tau)\langle u(\tau), u(t)\rangle_{W_{0}} d \tau \\
& \leq \frac{1}{2}\|u(t)\|_{W_{0}}^{2}+\frac{1}{2}\left\{\int_{0}^{t} g(t-\tau)\left(\|u(\tau)-u(t)\|_{W_{0}}+\|u(t)\|_{W_{0}}\right) d \tau\right\}^{2} \\
& \leq \frac{1}{2}\|u(t)\|_{W_{0}}^{2}+\frac{1}{2}(1+\eta)\left(\int_{0}^{t} g(t-\tau)\|u(t)\|_{W_{0}} d \tau\right)^{2} \\
& +\frac{1}{2}\left(1+\frac{1}{\eta}\right)\left(\int_{0}^{t} g(t-\tau)\|u(\tau)-u(t)\|_{W_{0}} d \tau\right)^{2} \\
& \leq \frac{1}{2}\|u(t)\|_{W_{0}}^{2}+\frac{1}{2}(1+\eta)(1-l)^{2}\|u(t)\|_{W_{0}}^{2}+\frac{1}{2}\left(1+\frac{1}{\eta}\right)(1-l)(g \diamond u)(t) .
\end{aligned}
$$

for any $\eta>0$. Now, letting $\eta=\frac{l}{1-l}>0$ then (3.37) yields

$$
\int_{0}^{t} g(t-\tau)\langle u(\tau), u(t)\rangle_{W_{0}} d \tau \leq \frac{2-l}{2}\|u(t)\|_{W_{0}}^{2}+\frac{1-l}{2 l}(g \diamond u)(t) .
$$

Substituting (3.38) into (3.33), we obtain

$$
\frac{d}{d t} F(t) \leq-\frac{1}{2}\left(\xi_{0}-\varepsilon \frac{1-l}{l}\right)(g \diamond u)(t)-\varepsilon\|u(t)\|_{W_{0}}^{2 \alpha}-\frac{\varepsilon l}{2}\|u(t)\|_{W_{0}}^{2}+\varepsilon \int_{\Omega}|u(t)|^{\rho-1} u(t) d x .
$$

Using the definition of $E(t)$ and (3.36) we have, for any positive constant $M$

$$
\begin{gathered}
\frac{d}{d t} F(t) \leq-M \varepsilon E(t)+\varepsilon\left(\frac{M}{2 \alpha}-1\right)\|u(t)\|_{W_{0}}^{2 \alpha}+\frac{\varepsilon}{2}\left[M+2 \theta l\left(1-\frac{M}{\rho}\right)-l\right]\|u(t)\|_{W_{0}}^{2} \\
+\frac{1}{2}\left[\varepsilon\left(\frac{1-l}{l}+\frac{M}{2}\right)-\xi_{0}\right](g \diamond u)(t) .
\end{gathered}
$$

At this point, we choose $1>M>0$ and $E(0)$ small sufficiently such that

$$
\frac{M}{2 \alpha}-1<0 \quad \text { and } \quad M+2 \theta l\left(1-\frac{M}{\rho}\right)-l<0 .
$$

After $M$ is fixed, we choose $\varepsilon$ small enough such that

$$
\varepsilon\left(\frac{1-l}{l}+\frac{M}{2}\right)-\xi_{0}<0 \text {. }
$$

Inequality (3.40) becomes

By (3.32), we have

$$
\frac{d}{d t} F(t) \leq-M \varepsilon E(t)
$$

$$
\frac{d}{d t} F(t) \leq-M \beta_{2} \varepsilon F(t)
$$

So $F(t) \leq C e^{-K t}$ where $K=M \beta_{2} \varepsilon>0$. Consequently, by using (3.32) once again, we conclude the result.

Thus, the proof of Theorem 3.3 is achieved. 
Acknowledgements. The authors wish to express their gratitude to the anonymous referee for reading the original manuscript carefully and making several suggestions. This work is partially supported by the Proyecto de Investigación $N^{0} 161401021$, UNMSM-FCM.

\section{References}

[1] Abdellaoui, B., Abdellaoui, B., Attar, A., Bentifour, R. et al., On fractional p-Laplacian parabolic problem with general data, Annali di Matematica Pura ed Applicata (2018), 197:329.

[2] Acerbi, E., Mingione, G., Regularity results for stationary electro-rheological fuids, Arch. Ration. Mech. Anal. 164(2002), 213-259.

[3] Alt, H.W., Di Benedetto, E., Nonsteady flow of water and oil through inhomogeneous porous media, Ann. Scuola Norm. Sup. Pisa Cl. Sci., 12(1985), no. 4, 335-392.

[4] Alves, C.O., Corrêa, F., Ma, T.F., Positive solutions for a quasilinear elliptic equation of Kirchhoff type, Comput. Math. Appl., 49(2005), 85-93.

[5] Andreu, F., Mazan,J.M., Rossi,J.D., Toledo,J., A nonlocal p-Laplacian evolution equationwith nonhomogeneous Dirichlet boundary conditions, SIAM J. Math. Anal., 40(2009), 1815-1851.

[6] Anello, G., A uniqueness result for a nonlocal equation of Kirchhoff type and some related open problems, J. Math. Anal. Appl., 373(2011), 248-251.

[7] Antontsev, S.N., Oliveira, H.B., Qualitative properties of the ice-thickness in a 3D model, WSEAS Trans. Math., 7(3)(2008), 78-86.

[8] Antontsev, S.N., Shmarev, S.I., A model porous medium equation with variable exponent of nonlinearity: existence, uniqueness and localization properties of solutions, Nonlinear Anal., 60(2005), 515-545.

[9] Antontsev, S.N., Shmarev, S.I., Simsen J., Simsen, M.S., On the evolution p-Laplacian with nonlocal memory, Nonlinear Anal., 134(2016), 31-54.

[10] Applebaum, D., Lévy process from probability to finance and quantum groups, Notices Amer. Math. Soc., 51(2004), 1336-1347.

[11] Autuori, G., Pucci, P., Salvatori, M.C., Asymptotic Stability for anisotropic Kirchhoff system, J. Math. Anal. Appl., 352(2009), 149-165.

[12] Barbu, V., Integro-differential equations in Hilbert spaces, An. Şti. Univ. "Al. I. Cuza" Iaşi, Secţia Mat. (N.S.), 19(1973), 365-383.

[13] Bellman, R., Inequalities, Springer-Verlag Berlin, Heidelgerg, New York, 1971.

[14] Blanchard, D., Francfort, G.A., Study of a doubly nonlinear heat equation with no growth assumptions on the parabolic term, SIAM J. Math. Anal., 19(5)(1988), 1032-1056.

[15] Bokalo, T.M., Some formulas of integration by parts in the spaces of functions with variable exponent of nonlinearity, Visn. L'viv. Univ., Ser. Mekh. Mat., 71(2009), 5-18.

[16] Bokalo, T.M., Buhrii, O.M., Doubly nonlinear parabolic equations with variable exponents of nonlinearity, Ukrainian Math. J., 63(2011), 709-728.

[17] Bucur, C., Valdinoci, E., Nonlocal diffusion and applications , Lecture Notes of the Unione Matematica Italiana, 20, Springer, [Cham], Unione Matematica Italiana, Bologna, 2016, xii+155 pp. 
[18] Chen, Y., Levine, S., Rao, M., Variable exponent, linear growth functionals in image restoration, SIAM J. Appl. Math., 66(2006), 1383-1406.

[19] Crandall, M.G., Londen S.O., Nohel, J.A., An abstract nonlinear Volterra integrodifferential equation, J. Math. Anal. Appl., 64(1978), 701-735.

[20] Daz, J., Talin, F., On a nonlinear parabolic problem arising in some models related to turbulent flows, SIAM J. Math. Anal., 25(1994), 1085-1111.

[21] Edmunds, D.E., Rákosník, J., Density of smooth functions in $W^{k, p(x)}(\Omega)$, Proc. R. Soc. A., 437(1992), 229-236.

[22] Edmunds, D.E., Rákosník, J., Sobolev embedding with variable exponent, Studia Math., 143(2000), 267-293.

[23] Fan, X.L., Shen, J.S., Zhao, D., Sobolev embedding theorems for spaces $W^{k ; p(x)}(\Omega)$, J. Math. Anal. Appl., 262(2001), 749-760.

[24] Fan, X.L., Zhang, Q.H., Existence of solutions for $p(x)$-Laplacian Dirichlet problems, Nonlinear Anal., 52(2003), 1843-1852.

[25] Fan, X.L., Zhao, D., On the Spaces $L^{p(x)}$ and $W^{m, p(x)}$, J. Math. Anal. Appl., 263(2001), 424-446.

[26] Fife, P., Some nonclassical trends in parabolic and parabolic-like evolutions, Trends in Nonlinear Analysis, Berlin, Springer, 2003, 153-91.

[27] Fiscella, A., Valdinoci, E., A critical Kirchhoff type problem involving a nonlocal operator, Nonlinear Anal., 94(2014), 156-170.

[28] Fu, Y., Pucci, P., On solutions of space-fractional diffusion equationsby means of potential wells, Electron. J. Qualitative Theory Differ. Equ., 70(2016),1-17.

[29] Giacomoni, J., Tiwari, S., Existence and global behavior of solutions to fractional pLaplacian parabolic problems, Electron. J. Diff. Equ., 3(2018), no. 44, 1-20.

[30] Gilardi, G., Stefanelli, U., Time-discretization and global solution for a doubly nonlinear Volterra equation, J. Differential Equations, 228(2006), 707-736.

[31] Gobbino, M., Quasilinear degenerate parabolic equation of Kirchhoff type, Math. Methods Appl. Sci., 22(1999), 375-388.

[32] He, X., Zou, W., Infinitely many positive solutions for Kirchhoff-type problems, Nonlinear Anal., 70(2009), no. 3, 1407-1414.

[33] Kalashnikov, A., Some problems of the qualitative theory of second-order nonlinear degenerate parabolic equations, Russian Math. Surveys, 42(1987), no. 2, 169-222.

[34] Kováčik, O., Rákosník, J., On the spaces $L^{p(x)}(\Omega)$ and $W^{1, p(x)}(\Omega)$, Czechoslovak Math. J., 41(1991), 592-618.

[35] Laskin, N., Fractional quantum mechanics and Lévy path integrals, Physics Letters A, 268(2000), 298-305.

[36] MacCamy, R.C., Stability theorems for a class of functional differential equations, SIAM J. Appl. Math., 30(1976), 557-576.

[37] Mao, A., Zhang, Z., Sign-changing and multiple solutions of Kirchhoff type problems without the P.S. condition, Nonlinear Anal.,70(2009), no. 3, 1275-1287.

[38] Mingqi, X., Rădulescu, V.,Zhang, B., Nonlocal Kirchhoff diffusion problems: local existence and blow-up of solutions, Nonlinearity, 31(2018), 3228-3250.

[39] Nguyen, H.-M., Squassina, M., Fractional Caffarelli-Kohn-Nirenberg inequalities, J. Funct. Anal., 274(2018), no. 9, 2661-2672. 
[40] Nohel, J., Nonlinear Volterra equations for heat flow in materials with memory, Technical Summary Report 2081, University of Wisconsin-Madison, 1980.

[41] Pan, N., Zhang, B., Cao, J., Degenerate Kirchhoff diffusion problems involving fractional p-Laplacian, Nonlin. Anal. RWA., 37(2017), no. 9, 56-70.

[42] Pucci, P., Xiang, M.Q., Zhang, B.L., A diffusion problem of Kirchhoff type involving the nonlocal fractional p-Laplacian, Discrete. Contin. Dyn. Syst., 37(2017), 4035-4051.

[43] Servadei, R., Valdinoci, E., Mountain Pass solutions for non-local elliptic operators, J. Math. Anal. Appl., 389(2012), 887-898.

[44] Servadei, R., Valdinoci, E., Variational methods for non-local operators of elliptic type, Discrete Contin. Dyn. Syst., 33(2013), 2105-2137.

[45] Servadei, R., Valdinoci, E., A Brezis-Nirenberg result for non-local critical equations in low dimension, Commun. Pure Appl. Anal., 12(2013), 2445-2464.

[46] Servadei, R., Valdinoci, E., The Brezis-Nirenberg result for the fractional Laplacian, Trans. Amer. Math. Soc., 367(2015), 67-102.

[47] Shin, K., Kang S., Doubly nonlinear Volterra equations involving the Leray-Lions operators, East Asian Math. J., 29(2013), 69-82.

[48] Stefanelli, U., Well-posedness and time discretization of a nonlinear Volterra integrodifferential equation, J. Integral Equations Appl., 13(2001), 273-304.

[49] Stefanelli, U., On some nonlocal evolution equations in Banach spaces, J. Evol. Equ., 4(2004), 1-26.

[50] Truong, L.X., Van, Y.N., On a class of nonlinear heat equations with viscoelastic term, Comp. Math. App., 72(2016), no. 1, 216-232.

[51] Valdinoci, E., From the long jump random walk to the fractional Laplacian, Bol. Soc. Esp. Mat. Apl. SMA, 49(2009), 33-44.

[52] Vazquez, C., Schiavi, E., Durany, J., Daz, J.I., Calvo, N., On a doubly nonlinear parabolic obstacle problem modelling ice sheet dynamics, SIAM J. Appl. Math., 63(2003), no. 2, 683-707.

[53] Zhikov, V.V., Solvability of the three-dimensional thermistor problem, Proc. Stekolov Inst. Math., 261(2008), 101-114.

Eugenio Cabanillas Lapa

Instituto de Investigación-FCM-UNMSM, Lima, Perú

e-mail: cleugenio@yahoo.com

Zacarias L. Huaringa Segura

Instituto de Investigación-FCM-UNMSM, Lima, Perú

e-mail: zhuaringas@unmsm.edu.pe

Juan B. Bernui Barros

Instituto de Investigación-FCM-UNMSM, Lima, Perú

e-mail: jbernuib@unmsm.edu.pe

Eduardo V. Trujillo Flores

Instituto de Investigación-FIARN-UNAC, Lima, Perú

e-mail: evtrujillof2005@yahoo.es 